\title{
Solvable Map Method for Integrating Nonlinear Hamiltonian Systems
}

\author{
Govindan Rangarajan and Minita Sachidanand \\ Department of Mathematics \\ Indian Institute of Science \\ Bangalore 560 012, India \\ rangaraj@math.iisc.ernet.in
}

\begin{abstract}
Conventional numerical integration algorithms can not be used for long term stability studies of complicated nonlinear Hamiltonian systems since they do not preserve the symplectic structure of the system. Further, they can be very slow even if supercomputers are used. In this paper, we study the symplectic integration algorithm using solvable maps which is both fast and accurate and extend it to six dimensions. This extension enables single particle studies using all three degrees of freedom.
\end{abstract}

\section{Introduction}

Consider a complicated Hamiltonian system that is non-integrable. Suppose we are interested in the long-term stability of this dynamical system. Since the system is assumed to be non-integrable, it is very difficult to give stability criteria in an analytic form. A possible solution is to numerically follow the trajectories of the particles through the system for a large number of iterations, a process which goes by the name tracking. One could then infer the stability of motion in the system by analyzing these tracking results. However, in long term integration of these systems, it is important to preserve the Hamiltonian nature of the system at every integration step. Otherwise, one can get spurious damping or even chaotic behaviour which is not present in the original system. Such problems becomes accentuated when long term integration is performed. This can then obviously lead to wrong predictions regarding the long-term stability of the Hamiltonian system being studied.

The most straightforward method that can be used to perform long term tracking is numerical integration. However, a very short time step has to be used to reduce the spurious behaviour that occurs because of the non-symplectic integration. This makes this method so slow that it is impractical to study the long term behaviour of very complicated systems (like the Large Hadron Collider) even if supercomputers are used. Therefore, we need a method that is both fast and accurate.

Several symplectic integration methods have been discussed in literature 112 , 3/4 5 6 678 8 9[10[1]. Methods using Lie algebraic perturbation theory which give

V.N. Alexandrov et al. (Eds.): ICCS 2001, LNCS 2073, pp. 894-903 2001.

(C) Springer-Verlag Berlin Heidelberg 2001 
maps where the final values of variables are explicit functions of initial ones offer several advantages. Further, since the whole complicated system can be represented by a single symplectic map, there can be a substantial saving in computation time as compared to non-map based methods. In this paper, we extend an alternate symplectic integration method using solvable maps [12 to six dimensions. This extension is necessary for single particle studies using all three degrees of freedom. The above method has been shown to give good results in lower dimensions 12 .

\section{Preliminaries}

Let $z=\left(q_{1}, q_{2}, \ldots, q_{n}, p_{1}, p_{2}, \ldots, p_{n}\right)$ denote the $2 n$ dimensional phase space variables. Denoting the initial and final locations of the particle by $z^{i}$ and $z^{f}$ respectively, the time evolution of a Hamiltonian system $H(z)$ can be described as a symplectic map $\mathcal{M}[13]$ :

$$
z^{f}=\mathcal{M} z^{i} .
$$

The symplectic map is related to the Hamiltonian $H(z)$ by the equation:

$$
\frac{d \mathcal{M}}{d t}=\mathcal{M}:-H\left(z_{0}\right),
$$

where $z_{0}$ is $z$ at time $t=0$.

Using the Dragt-Finn factorization theorem [13], the symplectic map $\mathcal{M}$ can be factorized as follows.

$$
\mathcal{M}=\hat{M} \exp \left(: f_{3}(z):\right) \cdots \exp \left(: f_{m}(z):\right) \cdots .
$$

Here $\hat{M}$ represents the linear part of the map and $f_{m}(z)$ is a homogeneous polynomial of degree $m$ in $z$ determined uniquely by the factorization theorem. Further : $f(z)$ : is the Lie operator corresponding to the function $f(z)$ and is defined by

$$
: f(z): g(z)=[f(z), g(z)]
$$

Here $g(z)$ denotes another phase space function and $[f(z), g(z)]$ denotes the usual Poisson bracket of the functions $f(z)$ and $g(z)$. The exponential of a Lie operator is called a Lie transformation and is given as

$$
\exp (: f(z):)=\sum_{n=0}^{\infty} \frac{: f(z):^{n}}{n !} .
$$

The map $\mathcal{M}$ involves an infinite number of Lie transformations and hence for any practical computation, we have to truncate $\mathcal{M}$ after a finite number of Lie transformations.

$$
\mathcal{M}=\hat{M} e^{: f_{3}:} e^{: f_{4}: \ldots} e^{: f_{m}:} .
$$

Each one of the Lie transformations in the above equation is a symplectic map and hence the map can be truncated at any order without losing symplecticity. 
The product $e^{: f_{3}:} e^{: f_{4}: \cdots e^{: f_{m}:}}$ in Eq. (6) give the nonlinear part of $\mathcal{M}$. Each of these Lie transformations gives an infinite number of terms when acting on phase space variables. Since we can not evaluate an infinite number of terms in any algorithm, we need to overcome this problem in some manner. The most straightforward method is to truncate the Taylor series expansion of each Lie transformation, after a finite number terms. This however violates the symplectic condition. Though this method is justifiable in short term tracking, it does not work well in long term tracking as the non-symplecticity can lead to spurious damping or even chaotic behaviour which is not present in the original system [10]. Therefore, we refactorize $\mathcal{M}$ in terms of simpler symplectic maps that can be evaluated both exactly and quickly. We achieve this refactorization through the so-called "solvable maps" [11,12].

\section{Solvable Map Method}

Solvable maps are generalizations of Cremona maps. The class of Cremona maps includes only those symplectic maps for which the Taylor series expansion terminates when acting on phase space coordinates. The class of solvable maps also includes those symplectic maps for which the Taylor series expansion can be summed up explicitly. One simple example of such a map is $\exp \left(: a q_{1}^{l+2}+b q_{1}^{l+1} p_{1}:\right)[12$.

The basic idea behind the solvable map method is to represent each nonlinear factor $\exp \left(: f_{m}:\right)$ in Eq. ([6) as a product of solvable maps, i.e.,

$$
\exp \left(: f_{m}:\right)=\exp \left(: h_{1}:\right) \exp \left(: h_{2}:\right) \cdots \exp \left(: h_{n}:\right) \text {, for } m \geq 3 \text {. }
$$

We also ensure that the number of solvable maps is a minimum. For simplicity, we restrict ourselves to a general fourth order symplectic map in six dimensions.

We first set up a notation that will facilitate the indexing of monomials in six phase space variables. Let $P_{j}$ denote the following basis monomial of degree $\mathrm{m}$ in six phase-space variables

$$
P_{j}^{(m)}=q_{1}^{r_{1}} p_{1}^{r_{2}} q_{2}^{r_{3}} p_{2}^{r_{4}} q_{3}^{r_{5}} p_{3}^{r_{6}}
$$

where $1 \leq r_{i} \leq m, \quad r_{1}+r_{2}+\ldots+r_{6}=m$. For this six dimensional case, the number of independent monomials of degree 3 is 56 and the number of independent monomials of degree 4 is 126 .

Consider a nonlinear Hamiltonian system in six phase space dimensions. Performing a Taylor expansion around the origin in the spirit of perturbation theory, the Hamiltonian $H(z)$ can be written as (up to fourth order in the phase space variables $z$ )

$$
H(z)=H_{2}(z)+H_{3}(z)+H_{4}(z) .
$$

Here $H_{m}(z)$ is a homogeneous polynomial of degree $m$ in $z$. Concentrating on the nonlinear part for the moment, $H_{3}$ and $H_{4}$ can be written as a linear combination 
of the basis monomials introduced above:

$$
\begin{aligned}
& H_{3}(z)=\gamma_{1} P_{1}(z)+\gamma_{2} P_{2}(z)+\cdots+\gamma_{56} P_{56}(z), \\
& H_{4}(z)=\gamma_{57} P_{57}(z)+\gamma_{58} P_{58}(z)+\cdots+\gamma_{182} P_{182}(z) .
\end{aligned}
$$

Now we express the time evolution of the Hamiltonian system $H(z)$ as a symplectic map $\mathcal{M}_{4}$ (truncated at order 4) using the Dragt-Finn factorization:

$$
\mathcal{M}_{4}=\hat{M} \exp \left(: f_{3}:\right) \exp \left(: f_{4}:\right),
$$

where $\hat{M}$ is obtained from the linear part $H_{2}(z)$ of the Hamiltonian $H(z)$. Using the basis monomials, $f_{3}$ and $f_{4}$ can be represented as:

$$
\begin{aligned}
& f_{3}(z)=\alpha_{1} P_{1}(z)+\alpha_{2} P_{2}(z)+\cdots+\alpha_{56} P_{56}(z), \\
& f_{4}(z)=\alpha_{57} P_{57}(z)+\alpha_{58} P_{58}(z)+\cdots+\alpha_{182} P_{182}(z) .
\end{aligned}
$$

It should be noted that there is a specific algorithm for determining the coefficients $\alpha_{i}$ in terms of the coefficients $\gamma_{i}$ and the linear part $H_{2}(z)$. For a specific Hamiltonian, many of the coefficients $\alpha_{i}$ could be zero.

We now refactorize the symplectic map $\mathcal{M}_{4}$ representing the original Hamiltonian system in terms of solvable maps as follows (for further details see [14]):

where

$$
\mathcal{M}=\hat{M} e^{: h_{1}:} e^{: h_{2}:} \ldots e^{: h_{20}:}
$$

$$
\begin{aligned}
h_{1}= & q_{1}^{4}\left[\beta_{57}\right]+q_{1}^{3}\left[\beta_{1}+\beta_{59} q_{2}+\beta_{61} q_{3}\right]+ \\
& q_{1}^{2}\left[\beta_{3} q_{2}+\beta_{5} q_{3}+\beta_{68} q_{2}^{2}+\beta_{70} q_{2} q_{3}+\beta_{75} q_{3}^{2}\right]+ \\
& q_{1}\left[\beta_{12} q_{2}^{2}+\beta_{14} q_{2} q_{3}+\beta_{19} q_{3}^{2}+\beta_{93} q_{2}^{3}+\beta_{95} q_{2}^{2} q_{3}+\beta_{100} q_{2} q_{3}^{2}+\right. \\
& \left.\beta_{109} q_{3}^{3}\right]+ \\
& {\left[\beta_{39} q_{2}^{2} q_{3}+\beta_{44} q_{2} q_{3}^{2}+\beta_{150} q_{2}^{3} q_{3}+\beta_{155} q_{2}^{2} q_{3}^{2}+\beta_{164} q_{2} q_{3}^{3}\right]+} \\
& p_{1}\left[\beta_{27} q_{2}^{2}+\beta_{29} q_{2} q_{3}+\beta_{34} q_{3}^{2}+\beta_{128} q_{2}^{3}+\beta_{130} q_{2}^{2} q_{3}+\right. \\
& \left.\beta_{135} q_{2} q_{3}^{2}+\beta_{144} q_{3}^{3}\right]+ \\
& q_{1} p_{1}\left[\beta_{8} q_{2}+\beta_{10} q_{3}+\beta_{83} q_{2}^{2}+\beta_{85} q_{2} q_{3}+\beta_{90} q_{3}^{2}\right] \\
h_{2}= & p_{1}^{4}\left[\beta_{113}\right]+p_{1}^{3}\left[\beta_{22}+\beta_{115} p_{2}+\beta_{117} p_{3}\right]+ \\
& p_{1}^{2}\left[\beta_{24} p_{2}+\beta_{26} p_{3}+\beta_{122} p_{2}^{2}+\beta_{124} p_{2} p_{3}+\beta_{127} p_{3}^{2}\right]+ \\
& p_{1}\left[\beta_{31} p_{2}^{2}+\beta_{33} p_{2} p_{3}+\beta_{36} p_{3}^{2}+\beta_{138} p_{2}^{3}+\beta_{140} p_{2}^{2} p_{3}+\right. \\
& \left.\beta_{143} p_{2} p_{3}^{2}+\beta_{147} p_{3}^{3}\right]+ \\
& {\left[\beta_{49} p_{2}^{2} p_{3}+\beta_{52} p_{2} p_{3}^{2}+\beta_{170} p_{2}^{3} p_{3}+\beta_{173} p_{2}^{2} p_{3}^{2}+\beta_{177} p_{2} p_{3}^{3}\right]+} \\
& q_{1}\left[\beta_{16} p_{2}^{2}+\beta_{18} p_{2} p_{3}+\beta_{21} p_{3}^{2}+\beta_{103} p_{2}^{3}+\beta_{105} p_{2}^{2} p_{3}+\right. \\
& \left.\beta_{108} p_{2} p_{3}^{2}+\beta_{112} p_{3}^{3}\right]+ \\
& q_{1} p_{1}\left[\beta_{9} p_{2}+\beta_{11} p_{3}+\beta_{87} p_{2}^{2}+\beta_{89} p_{2} p_{3}+\beta_{92} p_{3}^{2}\right] \\
h_{3}= & q_{2}^{4}\left[\beta_{148}\right]+q_{2}^{3}\left[\beta_{37}+\beta_{151} p_{3}\right]+ \\
& q_{2}^{2}\left[\beta_{40} p_{3}+\beta_{118} p_{1}^{2}+\beta_{131} p_{1} p_{3}+\beta_{157} p_{3}^{2}\right]+
\end{aligned}
$$




$$
\begin{aligned}
& q_{2}\left[\beta_{23} p_{1}^{2}+\beta_{30} p_{1} p_{3}+\beta_{46} p_{3}^{2}+\beta_{114} p_{1}^{3}+\beta_{121} p_{1}^{2} p_{3}+\right. \\
& \left.\beta_{137} p_{1} p_{3}^{2}+\beta_{167} p_{3}^{3}\right]+ \\
& q_{2} p_{2}\left[\beta_{28} p_{1}+\beta_{43} p_{3}+\beta_{119} p_{1}^{2}+\beta_{134} p_{1} p_{3}+\beta_{163} p_{3}^{2}\right] \text {, } \\
& \begin{aligned}
h_{4}= & p_{2}^{4}\left[\beta_{168}\right]+p_{2}^{3}\left[\beta_{47}+\beta_{169} q_{3}\right]+ \\
& p_{2}^{2}\left[\beta_{48} q_{3}+\beta_{72} q_{1}^{2}+\beta_{104} q_{1} q_{3}+\beta_{171} q_{3}^{2}\right]+
\end{aligned} \\
& p_{2}\left[\beta_{4} q_{1}^{2}+\beta_{17} q_{1} q_{3}+\beta_{50} q_{3}^{2}+\beta_{60} q_{1}^{3}+\beta_{73} q_{1}^{2} q_{3}+\beta_{106} q_{1} q_{3}^{2}+\beta_{174} q_{3}^{3}\right]+ \\
& q_{2} p_{2}\left[\beta_{13} q_{1}+\beta_{42} q_{3}+\beta_{69} q_{1}^{2}+\beta_{98} q_{1} q_{3}+\beta_{161} q_{3}^{2}\right] \text {, } \\
& h_{5}=q_{3}^{4}\left[\beta_{178}\right]+q_{3}^{3}\left[\beta_{53}\right]+q_{3}^{2}\left[\beta_{125} p_{1}^{2}+\beta_{141} p_{1} p_{2}\right]+ \\
& q_{3}\left[\beta_{25} p_{1}^{2}+\beta_{32} p_{1} p_{2}+\beta_{116} p_{1}^{3}+\beta_{123} p_{1}^{2} p_{2}+\beta_{139} p_{1} p_{2}^{2}\right]+ \\
& q_{3} p_{3}\left[\beta_{35} p_{1}+\beta_{51} p_{2}+\beta_{126} p_{1}^{2}+\beta_{142} p_{1} p_{2}+\beta_{172} p_{2}^{2}\right] \text {, } \\
& h_{6}=p_{3}^{4}\left[\beta_{182}\right]+p_{3}^{3}\left[\beta_{56}\right]+p_{3}^{2}\left[\beta_{77} q_{1}^{2}+\beta_{102} q_{1} q_{2}\right]+ \\
& p_{3}\left[\beta_{6} q_{1}^{2}+\beta_{15} q_{1} q_{2}+\beta_{62} q_{1}^{3}+\beta_{71} q_{1}^{2} q_{2}+\beta_{96} q_{1} q_{2}^{2}\right]+ \\
& q_{3} p_{3}\left[\beta_{20} q_{1}+\beta_{45} q_{2}+\beta_{76} q_{1}^{2}+\beta_{101} q_{1} q_{2}+\beta_{156} q_{2}^{2}\right] \text {, } \\
& h_{7}=\beta_{74} q_{1}^{2} p_{2} p_{3}, \\
& h_{8}=\beta_{120} p_{1}^{2} q_{2} q_{3}, \\
& h_{9}=q_{1}^{2} p_{1}\left[\beta_{2}+\beta_{64} q_{2}+\beta_{65} p_{2}+\beta_{66} q_{3}+\beta_{67} p_{3}\right], \\
& h_{10}=q_{1} p_{1}^{2}\left[\beta_{7}+\beta_{79} q_{2}+\beta_{80} p_{2}+\beta_{81} q_{3}+\beta_{82} p_{3}\right] \text {, } \\
& h_{11}=q_{2}^{2} p_{2}\left[\beta_{38}+\beta_{94} q_{1}+\beta_{129} p_{1}+\beta_{153} q_{3}+\beta_{154} p_{3}\right], \\
& h_{12}=q_{2} p_{2}^{2}\left[\beta_{41}+\beta_{97} q_{1}+\beta_{132} p_{1}+\beta_{159} q_{3}+\beta_{160} p_{3}\right], \\
& h_{13}=q_{3}^{2} p_{3}\left[\beta_{54}+\beta_{110} q_{1}+\beta_{145} p_{1}+\beta_{165} q_{2}+\beta_{175} p_{2}\right] \text {, } \\
& h_{14}=q_{3} p_{3}^{2}\left[\beta_{55}+\beta_{111} q_{1}+\beta_{146} p_{1}+\beta_{166} q_{2}+\beta_{176} p_{2}\right] \text {, } \\
& h_{15}=\beta_{58} q_{1}^{3} p_{1}+\beta_{149} q_{2}^{3} p_{2}+\beta_{179} q_{3}^{3} p_{3}, \\
& h_{16}=\beta_{78} q_{1} p_{1}^{3}+\beta_{158} q_{2} p_{2}^{3}+\beta_{181} q_{3} p_{3}^{3}, \\
& h_{17}=\beta_{86} q_{1} p_{1} q_{2} p_{3}+\beta_{88} q_{1} p_{1} p_{2} q_{3},
\end{aligned}
$$


$h_{18}=\beta_{99} q_{2} p_{2} q_{1} p_{3}+\beta_{133} q_{2} p_{2} p_{1} q_{3}$,

$h_{19}=\beta_{107} q_{3} p_{3} q_{1} p_{2}+\beta_{136} q_{3} p_{3} q_{2} p_{1}$,

$h_{20}=\beta_{63} q_{1}^{2} p_{1}^{2}+\beta_{84} q_{1} p_{1} q_{2} p_{2}+\beta_{91} q_{1} p_{1} q_{3} p_{3}+\beta_{152} q_{2}^{2} p_{2}^{2}+$ $\beta_{162} q_{2} p_{2} q_{3} p_{3}+\beta_{180} q_{3}^{2} p_{3}^{2}$.

Here $\beta_{i}$ 's are functions of $\alpha_{i}$ 's. They can be easily determined by comparing the original factorization for $\mathcal{M}_{4}$ in terms of $f_{i}$ with the solvable map refactorization in terms of $h_{i}$ order by order and using the Campbell-Baker-Hausdorff theorem [15]. Thus given $\gamma_{i}$ 's parameterizing the original Hamiltonian system $H(z), \beta_{i}$ 's can be readily determined [14.

We will now explicitly obtain the action of some of the above maps $e^{: h_{i}}$ : on the phase space variables in a closed form. This will also prove that these maps are solvable maps. A similar analysis can be done for the remaining maps 14. Consider the action of $e^{: h_{i}:}$ on $z$ :

$$
z^{\prime}=e^{: h_{i}:} z
$$

From Eq. (2) we see that this action is equivalent to first integrating the equations of motion for the Hamiltonian $h_{i}(z)$ from time $t=0$ to time $t=-1$ and then identifying the initial values $z_{0}($ at $t=0)$ with $z$ and the final values (at $t=-1$ ) for $z$ with $z^{\prime}$.

First, we consider the action of $e^{: h_{7}}$ on the phase space variables. Using the above procedure, this action can be easily obtained and is given as follows:

$$
\begin{array}{ll}
q_{1}^{\prime}=q_{1}, & p_{1}^{\prime}=p_{1}+2 \beta_{74} q_{1} p_{2} p_{3}, \\
q_{2}^{\prime}=q_{2}-\beta_{74} q_{1}^{2} p_{3}, & p_{2}^{\prime}=p_{2}, \\
q_{3}^{\prime}=q_{3}-\beta_{74} q_{1}^{2} p_{2}, & p_{3}^{\prime}=p_{3} .
\end{array}
$$

Thus, $e^{: h_{7}}$ is a solvable map. The action of the solvable map $e^{: h_{8}:}$ is similar to $e^{: h_{7}:}$.

The action of $e^{: h_{9}:}$ on the phase space variables is obtained as follows. The appropriate Hamiltonian is

$$
h_{9}=q_{1}^{2} p_{1}\left[\beta_{2}+\beta_{64} q_{2}+\beta_{65} p_{2}+\beta_{66} q_{3}+\beta_{67} p_{3}\right]=E_{9} .
$$

Here $E_{9}$ denotes the conserved numerical value of $h_{9}$ and is obtained by evaluating $h_{9}$ at $t=0$. We note that

$$
\frac{d}{d t}\left(q_{1}^{2} p_{1}\right)=2 q_{1} p_{1} \dot{q}_{1}+q_{1}^{2} \dot{p}_{1}=0 .
$$

Therefore, $q_{1}^{2} p_{1}=q_{1_{0}}^{2} p_{1_{0}}=$ constant . Consequently,

$$
\beta_{2}+\beta_{64} q_{2}+\beta_{65} p_{2}+\beta_{66} q_{3}+\beta_{67} p_{3}=\frac{E_{9}}{q_{1_{0}}^{2} p_{1_{0}}}=\text { constant. }
$$


Hamilton's equations of motion, give

$$
\dot{q}_{1}=\frac{\partial h_{9}}{\partial p_{1}}=q_{1}^{2}\left(\beta_{2}+\beta_{64} q_{2}+\beta_{65} p_{2}+\beta_{66} q_{3}+\beta_{67} p_{3}\right) .
$$

Integrating the above equation, we have

$$
q_{1}=\frac{q_{1_{0}}^{2} p_{1_{0}}}{q_{1_{0}} p_{1_{0}}-E_{9} t},
$$

where we have denoted the values of $q_{i}$ and $p_{i}$ at time $t=0$ by $q_{i_{0}}$ and $p_{i_{0}}$. Also, we will sometimes collectively denote $q_{1}, q_{2}, q_{3}$ by $q$ and $p_{1}, p_{2}, p_{3}$ by $p$. Further, if $q_{1_{0}}, p_{1_{0}} \neq 0$, we have $q_{1} \neq 0$ and from Eq. (17)

$$
\begin{aligned}
p_{1} & =\frac{E_{9}}{q_{1}^{2}\left(\beta_{2}+\beta_{64} q_{2}+\beta_{65} p_{2}+\beta_{66} q_{3}+\beta_{67} p_{3}\right)}, \\
& =p_{1_{0}}\left(\frac{q_{1_{0}}}{q_{1}}\right)^{2} .
\end{aligned}
$$

If $q_{1_{0}}=0$, then $q_{1}=q_{1_{0}}$ and $p_{1}=p_{1_{0}}$.

The equation for $q_{2}$ is given by

$$
\dot{q}_{2}=\frac{\partial h_{9}}{\partial p_{2}}=\beta_{65} q_{1_{0}}^{2} p_{1_{0}} .
$$

Once again integrating the above equation, we have

$$
q_{2}=q_{2_{0}}+\beta_{65} q_{1_{0}}^{2} p_{1_{0}} t .
$$

Similarly, integrating the equations of motion for the other variables and letting $(q, p) \rightarrow\left(q^{\prime}, p^{\prime}\right),\left(q_{0}, p_{0}\right) \rightarrow(q, p), t \rightarrow-1$, we get the action of $e^{: h_{9}:}$ on the phase space variables. Thus,

$$
\begin{aligned}
q_{1}^{\prime} & = \begin{cases}\frac{q_{1}^{2} p_{1}}{q_{1} p_{1}+E_{9}} & \text { if } \quad q_{1}, p_{1} \neq 0 \\
q_{1} & \text { otherwise },\end{cases} \\
p_{1}^{\prime} & = \begin{cases}p_{1}\left(\frac{q_{1}}{q_{1}^{\prime}}\right)^{2} & \text { if } q_{1}, p_{1} \neq 0 \\
p_{1} & \text { otherwise, }\end{cases} \\
q_{2}^{\prime} & =q_{2}-\beta_{65} q_{1}^{2} p_{1}, \\
p_{2}^{\prime} & =p_{2}+\beta_{64} q_{1}^{2} p_{1}, \\
q_{3}^{\prime} & =q_{3}-\beta_{67} q_{1}^{2} p_{1}, \\
p_{3}^{\prime} & =p_{3}+\beta_{66} q_{1}^{2} p_{1} .
\end{aligned}
$$

This shows that $e^{: h_{9}:}$ is a solvable map. Similarly, $e^{: h_{10}:}, e^{: h_{11}:}, \ldots, e^{: h_{14}:}$ can be shown to be solvable maps and their action on phase space variables can be got by appropriately changing $q$ and $p$ in the above equations. 
The action of the map $e^{: h_{15}:}$ on the phase space variables can be obtained in the following way. The corresponding Hamiltonian is

$$
h_{15}=\beta_{58} q_{1}^{3} p_{1}+\beta_{149} q_{2}^{3} p_{2}+\beta_{179} q_{3}^{3} p_{3} .
$$

From the equations of motion,

$$
\dot{q}_{1}=\frac{\partial h_{15}}{\partial p_{1}}=\beta_{58} q_{1}^{3} .
$$

Integrating, we have

$$
q_{1}=\frac{q_{1_{0}}}{\sqrt{1-2 \beta_{58} q_{1_{0}}^{2} t}} .
$$

Substituting (19) in the equation of motion for $p_{1}$ and integrating, we see that

$$
\dot{p}_{1}=-3 \beta_{58} q_{1}^{2} p_{1}, \quad \Rightarrow \quad p_{1}=p_{1_{0}}\left(1-2 \beta_{58} q_{1_{0}}^{2} t\right)^{3 / 2} .
$$

Similarly integrating the remaining equations of motion and letting $(q, p) \quad \rightarrow \quad\left(q^{\prime}, p^{\prime}\right)$, $\left(q_{0}, p_{0}\right) \rightarrow(q, p), t \rightarrow-1$, we get the action of $e^{: h_{15}:}$ on the phase space variables:

$$
\begin{aligned}
& q_{1}^{\prime}=\frac{q_{1}}{\sqrt{1+2 \beta_{58} q_{1}^{2}}}, \quad p_{1}^{\prime}=p_{1}\left(1+2 \beta_{58} q_{1}^{2}\right)^{3 / 2} \\
& q_{2}^{\prime}=\frac{q_{2}}{\sqrt{1+2 \beta_{149} q_{2}^{2}}}, \quad p_{2}^{\prime}=p_{2}\left(1+2 \beta_{149} q_{2}^{2}\right)^{3 / 2} \\
& q_{3}^{\prime}=\frac{q_{3}}{\sqrt{1+2 \beta_{179} q_{3}^{2}}}, \quad p_{3}^{\prime}=p_{3}\left(1+2 \beta_{179} q_{3}^{2}\right)^{3 / 2}
\end{aligned}
$$

Thus, $e^{: h_{15}:}$ is a solvable map. The action of the map $e^{: h_{16}:}$ is similar to that of $e^{: h_{15}:}$ and can be got by interchanging $q$ and $p$ and by letting $t \rightarrow-t$.

Finally, the action of the solvable map $e^{: h_{20}:}$ is as follows:

$$
\begin{aligned}
q_{1}^{\prime} & =q_{1} \exp \left[-\left(2 \beta_{63} q_{1} p_{1}+\beta_{84} q_{2} p_{2}+\beta_{91} q_{3} p_{3}\right)\right], \\
p_{1}^{\prime} & =p_{1} \exp \left[\left(2 \beta_{63} q_{1} p_{1}+\beta_{84} q_{2} p_{2}+\beta_{91} q_{3} p_{3}\right)\right], \\
q_{2}^{\prime} & =q_{2} \exp \left[-\left(\beta_{84} q_{1} p_{1}+2 \beta_{152} q_{2} p_{2}+\beta_{162} q_{3} p_{3}\right)\right], \\
p_{2}^{\prime} & =p_{2} \exp \left[\left(\beta_{84} q_{1} p_{1}+2 \beta_{152} q_{2} p_{2}+\beta_{162} q_{3} p_{3}\right)\right], \\
q_{3}^{\prime} & =q_{3} \exp \left[-\left(\beta_{91} q_{1} p_{1}+\beta_{162} q_{2} p_{2}+2 \beta_{182} q_{3} p_{3}\right)\right], \\
p_{3}^{\prime} & =p_{3} \exp \left[\left(\beta_{91} q_{1} p_{1}+\beta_{162} q_{2} p_{2}+2 \beta_{182} q_{3} p_{3}\right)\right] .
\end{aligned}
$$

Therefore $e^{: h_{20}:}$ is also a solvable map.

Using the above solvable map method, different nonlinear Hamiltonian systems were studied and good results were obtained. Since the results obtained are very similar to those already demonstrated in Ref. [12] for lower dimensions, the details are omitted here. 


\section{Conclusions}

We have studied a symplectic integration algorithm using solvable maps. The solvable map factorization for three degrees of freedom (up to order 4) was carried out and it involves 20 solvable maps. It has been shown [12] that this algorithm gives good results for various examples. It also provides a fast and accurate symplectic integration method for complicated Hamiltonian systems. Another advantage of this method is that it can be easily extended to higher dimensions since the computations involved are not that difficult when symbolic manipulation programs are used.

\section{Acknowledgements}

This work was supported by grants from DRDO and ISRO, India as part of funding for Nonlinear Studies Group. GR is also associated with the Centre for Theoretical Studies, Indian Institute of Science and is a honorary faculty member of the Jawaharlal Nehru Center for Advanced Scientific Research, Bangalore.

\section{References}

1. Sanz-Serna, J. M. and Calvo, M. P.: Numerical Hamiltonian Problems. Chapman \& Hall, London (1994) and references therein

2. Ruth, R. D.: A canonical integration technique. IEEE Trans. Nucl. Sci. 30 (1983) 2669-2671

3. Irwin, J.: A multi-kick factorization algorithm for nonlinear symplectic maps. SSC Report No. 228 (1989)

4. Rangarajan, G.: Invariants for symplectic maps and symplectic completion of symplectic jets. Ph.D Thesis. University of Maryland (1990)

5. Channell, P. J. and Scovel, C.: Symplectic integration of Hamiltonian systems. Nonlinearity 3 (1990) 231-259

6. Yoshida, H.: Construction of higher order symplectic integrators. Phys. Lett. A 150 (1990) 262-268

7. Rangarajan, G.: Symplectic completion of symplectic jets. J. Math. Phys. 37 (1996) 4514-4542. For associated group theoretical material, see Rangarajan, G.: Representations of $\mathrm{Sp}(6, \mathrm{R})$ and $\mathrm{SU}(3)$ carried by homogeneous polynomials. J. Math. Phys. 38 (1997) 2710-2719

8. Forest, E. and Ruth, R.: Fourth order symplectic integration. Physica D 43 (1990) 105-117

9. Dragt, A. J. and Abell, D. T.: Jolt factorization of symplectic maps. Int. J. Mod. Phys. 2B (1993) 1019

10. Rangarajan, G.: Jolt factorization of the pendulum map. J. Phys. A: Math. Gen. 31 (1998) 3649-3658

11. Rangarajan, G., Dragt, A. J. and Neri, F.: Solvable map representation of a nonlinear symplectic map. Part. Accel. 28 (1990) 119-124

12. Rangarajan, G. and Sachidanand, M.: Symplectic integration using solvable maps. J. Phys. A: Math. Gen. 33 (2000) 131-142 
13. Dragt, A. J.: Lectures on nonlinear orbit dynamics. In: Carrigan, R. A., Huson, F. and Month, M. (eds.): Physics of High Energy Particle Accelerators. American Institute of Physics, New York (1982) 147-313; Dragt, A. J., Neri, F., Rangarajan, G., Douglas, D. R., Healy, L. M. and Ryne, R. D.: Lie algebraic treatment of linear and nonlinear beam dynamics. Annu. Rev. Nucl. Part. Sci. 38 (1988) 455-496

14. Sachidanand, M.: Applications of Lie Algebraic Techniques to Hamiltonian Systems. Ph. D. Thesis. Indian Institute of Science (2000)

15. Cornwell, J. F.: Group Theory in Physics. Volume 1. Academic, London (1984) 